Bulletin of the Section of Logic

Volume 50/1 (2021), pp. 81-96

https://doi.org/10.18778/0138-0680.2020.20

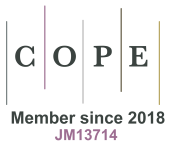

Ravikumar Bandaru*

Arsham Borumand Saeid

Young Bae Jun

\title{
ON GE-ALGEBRAS
}

\begin{abstract}
Hilbert algebras are important tools for certain investigations in intuitionistic logic and other non-classical logic and as a generalization of Hilbert algebra a new algebraic structure, called a GE-algebra (generalized exchange algebra), is introduced and studied its properties. We consider filters, upper sets and congruence kernels in a GE-algebra. We also characterize congruence kernels of transitive GE-algebras.
\end{abstract}

Keywords: (transitive) GE-algebra, filter, upper set, congruence kernel.

2010 Mathematical Subject Classification: 06F35, 03G25, 08A30.

\section{Introduction}

A. Monteiro in ([15]) (see also [16]) called Hilbert algebra a triple $(X, *, 1)$ where $X$ is a non-empty set, $*$ is a binary operation on $X, 1$ is an element of $X$ such that the following properties are satisfied for every $x, y, z \in X$ :

(H1) $x *(y * x)=1$

$(H 2)(x *(y * z)) *((x * y) *(x * z))=1$

${ }^{*}$ Corresponding author.

Presented by: Janusz Ciuciura

Received: September 16, 2019

Published online: August 30, 2020

(C) Copyright for this edition by Uniwersytet Łódzki, Łódź 2020 
(H3) $x * 1=1$

(H4) $x * y=1$ and $y * x=1$ imply $x=y$.

In 1960, L. Iturrioz proved that $(H 3)$ follows from $(H 1)$ and $(H 4)$ and that $(H 1),(H 2)$ and $(H 4)$ are independent. In the same year A. Diego, answering a problem posed by A. Monteiro, obtained an equational definition of these algebras. The literature on Hilbert algebras can be seen in ([8],[5, 4],[6],[11],[13],[10, 9]). Kim and Kim ([14]) introduced the notion of a BE-algebra as a generalization of a dual BCK-algebra. R. A. Borzooei and J. Shohani ([3]) introduced the notion of a generalized Hilbert algebra and studied its properties. J. C. Abbott ([1]) introduced a concept of implication algebra in the sake to formalize the logical connective implication in the classical propositional logic. R. A. Borzooei and S. K. Shoar ([2]) have shown that the implication algebras are equivalent to dual implicative BCK-algebras.

In this paper, we introduce the concept of GE-algebra which is a generalization of Hilbert algebra and study its properties. We define the notion of transitive and of commutative GE-algebra and observe that every commutative GE-algebra is a transitive GE-algebra. Also we give a condition under which a GE-algebra to become an Implication algebra. We give the relation between GE-algebra and other algebras (Hilbert algebra, dual implicative BCK-algebra, g-Hilbert algebra and BE-algebra). We consider filters, upper sets and congruence kernels in a GE-algebra and characterize congruence kernels whenever a GE-algebra is transitive.

\section{Preliminaries}

First, we recall certain definitions from $[1],[2],[5],[7],[12]$ and [14] that are required in the paper.

Definition 2.1. ([1]) An implication algebra is a set $X$ with a binary operation $*$ which satisfies the following conditions:

$\left(I_{1}\right)(x * y) * x=x$,

$\left(I_{2}\right)(x * y) * y=(y * x) * x$,

$\left(I_{3}\right) x *(y * z)=y *(x * z)$, for all $x, y, z \in X$. 
THEOREM 2.2. ([7]) In any implication algebra $(X, *)$, the following conditions hold:

(1) $x *(x * y)=x * y$.

(2) $x * x=y * y$.

(3) There exists a unique element 1 in $X$ such that

(a) $x * x=1,1 * x=x$ and $x * 1=1$.

(b) if $x * y=1$ and $y * x=1$, then $x=y$, for all $x, y \in X$.

Definition 2.3. ([2]) A dual BCK-algebra is a triple $(X, *, 1)$ where $\mathrm{X}$ is a non-empty set with a binary operation $*$ and a constant 1 satisfying the following axioms for all $x, y, z$ in $X$ :

$\left(D B C K_{1}\right)(y * z) *[(z * x) *(y * x)]=1$,

$\left(D B C K_{2}\right) y *[(y * x) * x]=1$,

$\left(D B K_{3}\right) x * x=1$,

$\left(D B C K_{4}\right) x * y=1$ and $y * x=1$ imply $x=y$,

$\left(\mathrm{DBCK}_{5}\right) x * 1=1$.

Definition 2.4. ([5]) A Hilbert algebra is an algebra $(X, *, 1)$ of type $(2,0)$ such that the following axioms hold, for all $x, y, z \in X$ :

(H1) $x *(y * x)=1$,

$(H 2)(x *(y * z)) *((x * y) *(x * z))=1$,

(H3) if $x * y=y * x=1$, then $x=y$.

It is proved that the above definition is equivalent to the system $\{H 4, H 5, H 6, H 7\}$ where:

(H4) $x * x=1$.

(H5) $1 * x=x$.

$(H 6) x *(y * z)=(x * y) *(x * z)$.

$(H 7)(x * y) *((y * x) * x)=(y * x) *((x * y) * y)$.

A Hilbert algebra $X$ is said to be commutative if it satisfies $\left(I_{2}\right)$. 
Definition 2.5. ([3]) A generalized Hilbert algebra (or biefly, g-Hilbert algebra) is an algebra $(X, *, 1)$ of type $(2,0)$ such that the following axioms hold, for all $x, y, z \in X$ :

$(G H 1) 1 * x=x$,

(GH2) $x * x=1$,

$(G H 3) x *(y * z)=y *(x * z)$,

$(G H 4) x *(y * z)=(x * y) *(x * z)$.

Definition 2.6. ([14]) A BE-algebra is an algebra $(X, *, 1)$ of type $(2,0)$ such that the following axioms hold, for all $x, y, z \in X$ :

$(B E 1) x * x=1$,

$(B E 2) 1 * x=1$,

$(B E 3) x * 1=1$,

$(B E 4) x *(y * z)=y *(x * z)$.

A BE-algebra $X$ is said to be commutative and self-distributive if it satisfies $\left(I_{2}\right)$ and $(H 6)$.

\section{GE-algebras}

In this section, we define the notion of a GE-algebra which is a generalization of the notion of Hilbert algebra and study its properties. Also, we define the notions of transitive GE-algebra and of commutative GE-algebra and give conditions under which a GE-algebra to become Implication algebra, dual implicative BCK-algebra and commutative Hilbert algebra.

Definition 3.1. A GE-algebra is a non-empty set $X$ with a constant 1 and a binary operation $*$ satisfying axioms:

$(G E 1) x * x=1$,

(GE2) $1 * x=x$,

$(G E 3) x *(y * z)=x *(y *(x * z))$, for all $x, y, z \in X$.

We can observe that every Hilbert algebra/implication algebra is a GEalgebra but the converse need not be true. 
Example 3.2. Let $X=\{1, a, b, c, d\}$ be a set with the following table.

\begin{tabular}{|c|c|c|c|c|c|}
\hline$*$ & 1 & $\mathrm{a}$ & $\mathrm{b}$ & $\mathrm{c}$ & $\mathrm{d}$ \\
\hline 1 & 1 & $\mathrm{a}$ & $\mathrm{b}$ & $\mathrm{c}$ & $\mathrm{d}$ \\
\hline $\mathrm{a}$ & 1 & 1 & $\mathrm{~b}$ & $\mathrm{~b}$ & 1 \\
\hline $\mathrm{b}$ & 1 & $\mathrm{a}$ & 1 & 1 & $\mathrm{~d}$ \\
\hline $\mathrm{c}$ & 1 & $\mathrm{a}$ & 1 & 1 & $\mathrm{~d}$ \\
\hline $\mathrm{d}$ & 1 & 1 & $\mathrm{c}$ & $\mathrm{c}$ & 1 \\
\hline
\end{tabular}

Then $(X, *, 1)$ is a GE-algebra but not a Hilbert algebra since $b * c=c * b=1$ but $b \neq c$.

Let $(X, *, 1)$ be a GE-algebra. Define a binary relation " $\leq$ " on $X$ by $x \leq y$ if and only if $x * y=1$.

There are no hidden difficulties to prove the following theorem hence we omit its proof.

THEOREM 3.3. In a GE-algebra $X$, for all $x, y, z \in X$, the following conditions hold:

(1) $x * 1=1$.

(2) $x *(x * y)=x * y$.

(3) $1 \leq x$ implies $x=1$.

(4) $x \leq y * x$.

(5) $x \leq(x * y) * y$.

(6) $x \leq(y * x) * x$.

(7) $x \leq(x * y) * x$.

(8) $x \leq y *(y * x)$.

(9) $x *(y * z) \leq y *(x * z)$.

(10) $x \leq y * z$ if and only if $y \leq x * z$.

The following theorem can be proved easily. 
Theorem 3.4. Let $(X, *, 1)$ be a GE-algebra. Then, for all $x, y, z \in X$, the following are equivalent.

(1) $x * y \leq(z * x) *(z * y)$,

(2) $x * y \leq(y * z) *(x * z)$.

Definition 3.5. A GE-algebra $(X, *, 1)$ is said to be transitive if it satisfies

$$
x * y \leq(z * x) *(z * y)
$$

for all $x, y, z \in X$.

Example 3.6. Let $X=\{1, a, b, c, d\}$ be a set with the following table.

\begin{tabular}{|c|c|c|c|c|c|}
\hline$*$ & 1 & $\mathrm{a}$ & $\mathrm{b}$ & $\mathrm{c}$ & $\mathrm{d}$ \\
\hline $\mathrm{1}$ & 1 & $\mathrm{a}$ & $\mathrm{b}$ & $\mathrm{c}$ & $\mathrm{d}$ \\
\hline $\mathrm{a}$ & 1 & 1 & 1 & $\mathrm{c}$ & $\mathrm{c}$ \\
\hline $\mathrm{b}$ & 1 & $\mathrm{a}$ & 1 & $\mathrm{~d}$ & $\mathrm{~d}$ \\
\hline $\mathrm{c}$ & 1 & $\mathrm{a}$ & 1 & 1 & 1 \\
\hline $\mathrm{d}$ & 1 & $\mathrm{a}$ & 1 & 1 & 1 \\
\hline
\end{tabular}

Then $(X, *, 1)$ is a transitive GE-algebra but not Hilbert algebra/dual BCK-algebra/BE-algebra since $a *(b * c)=a * d=c \neq d=b * c=b *(a * c)$.

The following theorem can be proved easily.

TheOrem 3.7. In a transitive GE-algebra $\left(X,{ }^{*}, 1\right)$, for all $x, y, z \in X$, the following conditions hold:

(1) $x \leq y$ implies $z * x \leq z * y$.

(2) $x * y \leq(y * z) *(x * z)$.

(3) $x \leq y$ implies $y * z \leq x * z$.

(4) $((x * y) * y) * z \leq x * z$.

(5) $x \leq y$ and $y \leq z$ imply $x \leq z$.

Definition 3.8. A GE-algebra $(X, *, 1)$ is said to be commutative if it satisfies $(x * y) * y=(y * x) * x$, for all $x, y \in X$. 
We can observe that every commutative GE-algebra is a transitive GEalgebra. But converse need not be true. From Example 3.6, we can observe that

$$
(b * c) * c=1 * c=c \neq b=1 * b=(c * b) * b .
$$

The following theorem shows that every commutative GE-algebra is a Hilbert algebra.

Theorem 3.9. If $(X, *, 1)$ is a commutative GE-algebra then $X$ is a Hilbert algebra.

Proof: Let $X$ be a commutative GE-algebra and $x, y, z \in X$. Then

(i) $x *(y * x)=x *(y *(x * x))=x *(y * 1)=x * 1=1$. (ii) Let $x * y=1$ and $y * x=1$. Then $(x * y) * y=y$ and hence $(y * x) * x=x$ which implies $x=y$. (iii) We know that $x *(y * z)=y *(x * z) \leq(x * y) *(x *(x * z))=(x * y) *(x * z)$. Hence $(x *(y * z)) *[(x * y) *(x * z)]=1$. Thus $X$ is a Hilbert algebra

The converse of the above theorem need not be true.

Example 3.10. Let $X=\{1, a, b, c\}$ be a set with the following table.

\begin{tabular}{|c|c|c|c|c|}
\hline$*$ & 1 & $\mathrm{a}$ & $\mathrm{b}$ & $\mathrm{c}$ \\
\hline 1 & 1 & $\mathrm{a}$ & $\mathrm{b}$ & $\mathrm{c}$ \\
\hline $\mathrm{a}$ & 1 & 1 & 1 & 1 \\
\hline $\mathrm{b}$ & 1 & $\mathrm{a}$ & 1 & 1 \\
\hline $\mathrm{c}$ & 1 & $\mathrm{a}$ & $\mathrm{b}$ & 1 \\
\hline
\end{tabular}

Then $(X, *, 1)$ is a Hilbert algebra which is not a commutative GE-algebra, since

$$
(a * b) * b=1 * b=b \neq 1=a * a=(b * a) * a .
$$

Theorem 3.11. Every generalized Hilbert algebra is a GE-algebra.

Proof: Let $(X, *, 1)$ be a generalized Hilbert algebra and $x, y, z \in X$. Then $x *(y *(x * z))=y *(x *(x * z))=y *((x * x) *(x * z))=y *(1 *(x * z))=$ $y *(x * z)=x *(y * z)$. Hence $(X, *, 1)$ is a GE-algebra.

The converse of the above theorem need not be true. From Example 3.2 , we can observe that $X$ is a GE-algebra but not a generalized Hilbert algebra since

$$
d *(a * b)=d * b=c \neq b=a * c=a *(d * b) .
$$


THEOREM 3.12. Every commutative GE-algebra is a generalized Hilbert algebra.

Proof: Let $(X, *, 1)$ be a commutative GE-algebra. Then $X$ is a Hilbert algebra and hence a generalized Hilbert algebra.

The converse of the above theorem need not be true. From Example 3.10 , we can observe that $X$ is a generalized Hilbert algebra but not a commutative GE-algebra.

TheOrem 3.13. Every self-distributive BE-algebra is a GE-algebra.

Proof: Let $(X, *, 1)$ be a self-distributive BE-algebra and $x, y, z \in X$. Then $x *(x * y)=(x * x) *(x * y)=1 *(x * y)=x * y$ and $x *(y * z)=$ $x *(x *(y * z))=x *(y *(x * z))$. Hence $X$ is a GE-algebra.

The converse of the above theorem need not be true. From Example 3.2 , we can observe that $X$ is a GE-algebra but not a self-distributive BE-algebra.

Theorem 3.14. Let $(X, *, 1)$ be a BE-algebra satisfying the property $x *$ $(x * y)=x * y$, for all $x, y \in X$. Then $X$ is a GE-algebra.

All non-existential results known for BE-algebras apply to GE-algebras. Theorem 3.15. Let $X$ be a GE-algebra. Then $X$ is commutative if and only if $X$ is an implication algebra.

Example 3.16. Let $X=\{1, a, b, c\}$ be a set with the following table.

\begin{tabular}{|c|c|c|c|c|}
\hline$*$ & 1 & $\mathrm{a}$ & $\mathrm{b}$ & $\mathrm{c}$ \\
\hline 1 & 1 & $\mathrm{a}$ & $\mathrm{b}$ & $\mathrm{c}$ \\
\hline $\mathrm{a}$ & 1 & 1 & 1 & 1 \\
\hline $\mathrm{b}$ & 1 & 1 & 1 & 1 \\
\hline $\mathrm{c}$ & 1 & 1 & 1 & 1 \\
\hline
\end{tabular}

Then $(X, *, 1)$ is a GE-algebra which is not an Implication algebra, since $(b * c) * c \neq(c * b) * b$. Hence, a commutative condition is necessary in the last theorem. 
Proposition 3.17. Let $(X, *, 1)$ be a GE-algebra. Then the following are equivalent.

(i) $X$ is commutative,

(ii) $X$ is implication algebra,

(iii) $X$ is dual implicative BCK-algebra,

(iv) $X$ is commutative Hilbert algebra.

\section{Filters and upper sets}

In this section, we introduce filters and upper sets in a GE-algebra and study their properties. We characterize filters in terms of upper sets.

Definition 4.1. A subset $F$ of $X$ is called a filter of $X$ if it satisfies the following:

$(G E F 1) 1 \in F$

$(G E F 2)$ if $x * y \in F$ and $x \in F$ then $y \in F$.

Obviously, $\{1\}$ and $X$ are filters of $X$. A filter $F$ is said to be proper if $F \neq X$.

Example 4.2.

(a) In Example 3.2, we can see that $I_{1}=\{1, a, d\}$ and $I_{2}=\{1, b, c\}$ are filters of $X$.

(b) In Example 3.6, we can see that $I_{1}=\{1, a\}, I_{2}=\{1, a, d\}, I_{3}=$ $\{1, b, c\}$ and $I_{4}=\{1, d\}$ are filters of $X$.

We denote the set of all filters of $X$ by $F(X)$.

The proof of the following lemma is straightforward and hence we omit the proof.

LEMma 4.3. If $\left\{F_{i}\right\}_{i \in \Lambda}$ is a family of filters of $X$, then $\bigcap_{i \in \Lambda} F_{i}$ is a filter of $X$.

Since the set $F(X)$ is closed under arbitrary intersections, we have the following theorem. 
TheOrem 4.4. $(F(X), \subseteq)$ is a complete lattice.

Proposition 4.5. Let $F$ be a filter of $X$. If $a \in F$ and $a \leq x$, then $x \in F$.

TheOrem 4.6. Let $X$ be a GE-algebra and $F$ a non-empty subset of $X$ satisfying the following conditions:

$(G E F 3) x \in X$ and $y \in F$ imply $x * y \in F$

(GEF4) $x \in X, a, b \in F$ imply $(a *(b * x)) * x \in F$.

Then $F$ is a filter of $X$.

Proof: Let $F$ be a non-empty subset of $X$ satisfying (GEF3) and (GEF4). Then $1 \in F$. Hence $(G E F 1)$ holds. Let $x \in F$ and $x * y \in F$. Then $y=1 * y=[(x * y) *(x * y)] * y \in F$ and hence $(G E F 2)$ holds. Therefore $F$ is a filter of $X$.

THEOREM 4.7. If $X$ is a GE-algebra and $F$ is a filter of $X$, then $F$ satisfies (GEF3) and (GEF4).

Proof: Let $F$ be a filter of $X$ and $a \in F, x \in X$. Then $a *(x * a)=1 \in F$ and hence, by $(G E F 2), x * a \in F$. Let $a, b \in F$. Since $a *[(a *(b * x)) *(b * x)]=$ $1 \in F$, we have $(a *(b * x)) *(b * x) \in F$. Hence $b *[(a *(b * x)) * x]=$ $b *[(a *(b * x)) *(b * x)] \in F$. Thus $(a *(b * x)) * x \in F$.

Theorem 4.8. Let $F$ be a non-empty subset $X$. Then $F$ is a filter of $X$ if and only if for every $a, b \in F$ and $x \in X, a *(b * x)=1$ implies $x \in F$.

Proof: Suppose $F$ is a filter of $X$ and $a, b \in F, x \in X$ such that $a *(b * x)=$ 1. By $(G E F 1)$, we have $a *(b * x) \in F$. Then, by $(G E F 2)$, we obtain $x \in F$. Conversely, assume that the condition holds. Let $a \in F$. Then $a *(a * 1)=1$ implies $1 \in F$. Suppose $x * y \in F$ and $x \in F$. Then $x *[(x * y) * y]=1$ implies $y \in F$. Hence $F$ is a filter of $X$.

Corollary 4.9. Let $F$ be a non-empty subset of $X$. Then $F$ is a filter of $X$ if and only if for every $a_{i} \in F(i \in \mathbb{N})$ and $x \in X, a_{n} *\left(\cdots *\left(a_{1} * x\right) \cdots\right)=1$ implies $x \in F$.

Lemma 4.10. Let $F$ be a filter of $X$. Then $(a * x) * x \in F$ for all $a \in F$ and $x \in X$.

Proposition 4.11. A non-empty subset $F$ of a GE-algebra $X$ is a filter of $X$ if and only if it satisfies $(i) 1 \in F(i i) x *(y * z) \in F, y \in F$ implies $x * z \in F$ for all $x, y, z \in X$. 
Let $X$ be a GE-algebra and $x, y \in X$. Define

$$
U(x)=\{z \in X \mid x * z=1\} \text { and } U(x, y)=\{z \in \mid x *(y * z)=1\}
$$

The set $U(x)$ (resp. $U(x, y))$ is called an upper set of $x$ (resp. of $x$ and $y$ ). We can observe that $1, x \in U(x)$ and $1, x, y \in U(x, y)$. Also, $U(1)=\{1\}$ is always a filter of $X$.

The following theorem can be proved easily.

Theorem 4.12. Let $X$ be a GE-algebra. Then, for any $x, y \in X$,

(i) $U(x, y)$ is a subalgebra of $X$.

(ii) $U(x)=\bigcap_{y \in X} U(x, y)$.

(iii) $U(x, y)=U(y, x)$.

Corollary 4.13. Let $F$ be a non-empty subset of $X$. Then $F$ is a filter of $X$ if and only if $U(x, y) \subseteq F$ for all $x, y \in F$.

Proof: Let $F$ be a filter of $X$ and $x, y \in F, z \in U(x, y)$. Then $x *(y * z)=$ $1 \in F$ and hence $z \in F$. So that $U(x, y) \subseteq F$. Conversely, assume that $U(x, y) \subseteq F$ for all $x, y \in F$. Since $F$ is non-empty, we have $z \in F$ such that $1 \in U(z, z) \subseteq F$. Hence $(G E F 1)$ holds. Let $x * y \in F$ and $x \in F$. Then $y \in U(x * y, x) \subseteq F$. Thus $(G E F 2)$ holds. Therefore $F$ is a filter of $X$.

Proposition 4.14. Let $X$ be a GE-algebra and $F$ a filter of $X$. Then, for any $x, y \in F$,

(i) $U(x) \subseteq F$.

(ii) $F=\bigcup_{x, y \in F} U(x, y)$.

Theorem 4.15. Let $X$ be a transitive GE-algebra and $x, y \in X$. Then, for any $x, y \in X$,

(i) $U(x, y)$ is a filter of $X$.

(ii) $U(x)$ is a filter of $X$. 
(iii) $x \leq y$ if and only if $U(y) \subseteq U(x)$.

(iv) $x \leq y$ and $y \leq x$ if and only if $U(x)=U(y)$.

Finally, we conclude this section with the following theorem.

TheOrem 4.16. Let $X$ be a transitive GE-algebra and $x, y \in X$. Then $y \in U(x)$ if and only if $U(x)=U(x, y)$.

\section{Congruence kernels}

In this section, we give a characterization of congruence kernels in a transitive GE-algebra. Let $\theta$ be a binary relation on a $\operatorname{GE}$-algebra $(X, *, 1)$. We denote $\{x \in X \mid(x, 1) \in \theta\}$ by $[1]_{\theta}$. If $\theta$ is a congruence relation on $X$ then $[1]_{\theta}$ is called a congruence kernel.

LEMMA 5.1. If $\theta$ is a congruence relation on $X$ then kernel $[1]_{\theta}$ is a filter of $X$.

Proof: Clearly $1 \in[1]_{\theta}$. Suppose $x \in[1]_{\theta}$ and $x * y \in[1]_{\theta}$. Then $(x, 1),(x * y, 1) \in \theta$ and hence $(x * y, y)=(x * y, 1 * y) \in \theta$. By symmetry of $\theta,(y, x * y) \in \theta$. Therefore, by transitivity of $\theta$, we obtain $(y, 1) \in \theta$ proving $y \in[1]_{\theta}$.

TheOrem 5.2. Let $(X, *, 1)$ be a transitive GE-algebra. Then every filter $F$ of $X$ is a kernel of a congruence $\theta_{F}$ given by

$$
(x, y) \in \theta_{F} \text { if and only if } x * y \in F \text { and } y * x \in F .
$$

Moreover, $\theta_{F}$ is the greatest congruence on $X$ having the kernel $F$.

Proof: Let $F$ be a filter of $X$. Since $1 \in F$, we have $\theta_{F}$ is reflexive. Clearly $\theta_{F}$ is symmetric. We prove transitivity of $\theta_{F}$. Let $(x, y) \in \theta_{F}$ and $(y, z) \in \theta_{F}$. Then $x * y, y * x, y * z, z * y \in F$. Hence, by Theorem $3.7(2)$ and by Proposition 4.5, $(y * z) *(x * z) \in F$. Therefore $x * z \in F$. Similarly, we can prove that $z * x \in F$. Thus $(x, z) \in \theta_{F}$. Now, we prove the substitution property of $\theta_{F}$. Let $(x, y) \in \theta_{F}$ and $(u, v) \in \theta_{F}$. Then $x * y, y * x, u * v, v * u \in F$ and hence, by Theorem 3.7(2) and by Proposition $4.5,(x * u) *(y * u) \in F$ and $(y * u) *(x * u) \in F$. Therefore, $(x * u, y * u) \in \theta_{F}$. Since $X$ is transitive, we have, by Proposition 4.5, $(y * u) *(y * v) \in F$ and 
$(y * v) *(y * u) \in F$. Hence $(y * u, y * v) \in \theta_{F}$. By transitivity of $\theta_{F}$, we conclude $(x * u, y * v) \in \theta_{F}$. Thus $\theta_{F}$ is a congruence relation on $X$.

If $x \in F$ then $1 * x=x \in F$ and $x * 1=1 \in F$. Therefore $(x, 1) \in \theta_{F}$, i.e., $x \in[1]_{\theta_{F}}$. Conversely, let $x \in[1]_{\theta_{F}}$. Then $(x, 1) \in \theta_{F}$ and hence $x=1 * x \in F$ which shows that $F=[1]_{\theta_{F}}$. Thus $F$ is the kernel of the congruence $\theta_{F}$.

Finally, if $\psi$ is a congruence relation on $X$ such that $[1]_{\psi}=F$ then for $(x, y) \in \psi$ we have $(x * y, 1)=(x * y, y * y) \in \psi$ and $(y * x, 1)=(y * x, y * y) \in \psi$ thus $x * y \in F$ and $y * x \in F$ which gives $(x, y) \in \theta_{F}$. Hence $\psi \subseteq \theta_{F}$ i.e., $\theta_{F}$ is the greatest congruence relation of $X$ having the kernel $F$.

The following example shows that filters need not be congruence kernels in a GE-algebra

Example 5.3. Let $X=\{1, a, b, c\}$ be a set with the following table.

\begin{tabular}{|c|c|c|c|c|}
\hline$*$ & 1 & $\mathrm{a}$ & $\mathrm{b}$ & $\mathrm{c}$ \\
\hline 1 & 1 & $\mathrm{a}$ & $\mathrm{b}$ & $\mathrm{c}$ \\
\hline $\mathrm{a}$ & 1 & 1 & 1 & $\mathrm{c}$ \\
\hline $\mathrm{b}$ & 1 & $\mathrm{a}$ & 1 & 1 \\
\hline $\mathrm{c}$ & 1 & $\mathrm{a}$ & $\mathrm{b}$ & 1 \\
\hline
\end{tabular}

Then $(X, *, 1)$ is a GE-algebra. But it is not transitive since $(b * c) *[(a * b) *$ $(a * c)]=c \neq 1$. Clearly, $F=\{1, a, b\}$ is a filter of $X$. Let $(b, a) \in \theta$ for some congruence relation $\theta$ on $X$. Then $(1, c) \in \theta$ and hence $c \in[1]_{\theta} \neq\{1, a, b\}$. Thus $F$ is not a congruence kernel.

Finally, we conclude this section with the following theorem.

Theorem 5.4. Let $(X, *, 1)$ be a transitive GE-algebra. Then filters of $X$ coincide with congruence kernels.

\section{Conclusion and future work}

Hilbert algebras represent the algebraic counterpart of the implicative fragment of intuitionistic propositional logic. In fact, Hilbert algebras are an algebraic counterpart of positive implicational calculus. Various type of generalization of algebraic structures were defined in the literature.

In this paper, we have introduced the concept of GE-algebras as a generalization of Hilbert algebras and studied their properties. In addition, we 
have considered filters and upper sets in a GE-algebra and characterized filters in terms of upper sets. We characterized congruence kernels in a transitive GE-algebra. Finally, we show that filters and congruence kernels coincide in a transitive GE-algebra.

We hope this work would serve as a foundation for further studies on the structure of GE-algebras like fuzzy GE-algebras, soft GE-algebras and hyper GE-algebras.

Acknowledgements. The authors are grateful to the referee for his valuable comments and suggestions for the improvement of the paper.

\section{References}

[1] J. C. Abbott, Semi-boolean algebra, Matematički Vesnik, vol. 4(19) (1967), pp. 177-198.

[2] R. A. Borzooei, S. Khosravi Shoar, Implication algebras are equivalent to the dual implicative BCK-algebras, Scientiae Mathematicae Japonicae, vol. 63(3) (2006), pp. 429-431.

[3] R. A. Borzooei, J. Shohani, On generalized Hilbert algebras, Italian Journal of Pure and Applied Mathematics, vol. 29 (2012), pp. 71-86.

[4] D. Buşneag, A note on deductive systems of a Hilbert algebra, Kobe Journal of Mathematics, vol. 2(1) (1985), pp. 29-35.

[5] D. Buşneag, Categories of algebraic logic, Editura Academiei Romane (2006).

[6] S. Celani, A note on homomorphisms of Hilbert algebras, International Journal of Mathematics and Mathematical Sciences, vol. 29(1) (2002), pp. 55-61, DOI: https://doi.org/10.1155/S0161171202011134.

[7] W. Y. C. Chen, J. S. Oliveira, Implication algebras and the MetropolisRota axioms for cubic lattices, Journal of Algebra, vol. 171(2) (1995), pp. 383-396, DOI: https://doi.org/10.1006/jabr.1995.1017.

[8] A. Diego, Sur les algèbres de Hilbert, Translated from the Spanish by Luisa Iturrioz. Collection de Logique Mathématique, Sér. A, Fasc. XXI, Gauthier-Villars, Paris; E. Nauwelaerts, Louvain (1966).

[9] A. Figallo, Jr., A. Ziliani, Remarks on Hertz algebras and implicative semilattices, Bulletin of the Section of Logic, vol. 34(1) (2005), pp. 37-42. 
[10] A. V. Figallo, G. Z. Ramón, S. Saad, A note on the Hilbert algebras with infimum, Matemática Contemporânea, vol. 24 (2003), pp. 23-37, 8th Workshop on Logic, Language, Informations and Computation - WoLLIC'2001 (Brasília).

[11] S. M. Hong, Y. B. Jun, On deductive systems of Hilbert algebras, Korean Mathematical Society. Communications, vol. 11(3) (1996), pp. 595-600.

[12] Y. Imai, K. Iséki, On axiom systems of propositional calculi, XIV, Proceedings of the Japan Academy, vol. 42(1) (1966), pp. 19-22, DOI: https://doi.org/10.3792/pja/1195522169.

[13] Y. B. Jun, Commutative Hilbert algebras, Soochow Journal of Mathematics, vol. 22(4) (1996), pp. 477-484.

[14] H. S. Kim, Y. H. Kim, On BE-algebras, Scientiae Mathematicae Japonicae, vol. 66(1) (2007), pp. 113-116.

[15] A. Monteiro, Lectures on Hilbert and Tarski Algebras, Instituto de Matemática, Universidad Nacional del Sur, Bahía Blanca, Argentina (1960).

[16] A. A. Monteiro, Sur les algèbres de Heyting symétriques, Portugaliae Mathematica, vol. 39(1-4) (1980), pp. 1-237 (1985), special issue in honor of António Monteiro.

\section{Ravikumar Bandaru}

GITAM (Deemed to be University)

Department of Mathematics

Hyderabad Campus

Telangana-502329, India

e-mail: ravimaths83@gmail.com

\section{Arsham Borumand Saeid}

Shahid Bahonar University of Kerman

Department of Pure Mathematics

Faculty of Mathematics and Computer

Kerman, Iran

e-mail: arsham@uk.ac.ir 
96 Ravikumar Bandaru, Arsham Borumand Saeid, Young Bae Jun

Young Bae Jun

Gyeongsang National University

Department of Mathematics Educations

Jinju 52828, Korea

e-mail: skywine@gmail.com 